\title{
Otopalatodigital syndrome spectrum disorders: otopalatodigital syndrome types 1 and 2, frontometaphyseal dysplasia and Melnick-Needles syndrome
}

The term otopalatodigital syndrome spectrum disorders is an umbrella category that includes four phenotypically related conditions, otopalatodigital syndrome types 1 and 2, frontometaphyseal dysplasia and Melnick-Needles syndrome. The phenotype of these conditions in the male ranges from a severe perinatally lethal multiple malformation syndrome to a mild skeletal dysplasia. Most, but not all, instances of these conditions are associated by mutations in the X-linked gene encoding the cytoskeletal protein filamin A. Mutations in this gene are clustered, exhibit a strong genotype-phenotype correlation and are presumed to exert their effect by a gain-of-function mechanism.

\section{In brief}

- Otopalatodigital syndrome types 1 and 2, frontometaphyseal dysplasia and Melnick-Needles syndrome are skeletal dysplasias characterized by anomalous ossification and skeletal patterning of the axial and appendicular skeleton.

- Extra skeletal malformations are also observed and include hydrocephalus, encephalocoele, cleft palate, cardiac defects, omphalocoele and obstructive uropathy.

- Most pedigrees are consistent with X-linked inheritance. Male-to-male transmission has not been reported.

- Mutations in the gene FLNA have been reported in the majority of cases of FMD and OPD2. All instances of typical OPD1 and MNS have mutations in FLNA.

- The phenotypes associated with mutations in FLNA are allelic to periventricular nodular heterotopia, a neuronal migration disorder characterized by seizures, mild cognitive disability and periventricular heterotopic neurones abutting the walls of the lateral cerebral ventricles.

- Mutations in FLNA that underlie the otopalatodigital syndrome spectrum conditions are all missense or small, in-frame deletions, that are predicted to preserve the translational reading frame and are presumed to exert a gain-of-function effect.

- Mutations underlying the otopalatodigital syndrome spectrum disorders are clustered and exhibit some genotype-phenotype correlation.
Stephen P Robertson*,

${ }^{1}$ Department of Paediatrics and Child Health, Dunedin School of Medicine, Otago University, Dunedin, New Zealand

European Journal of Human Genetics (2007) 15, 3-9. doi:10.1038/sj.ejhg.5201654; published online 23 August 2006

Keywords: otopalatodigital syndrome 1; otopalatodigital syndrome type 2; Melnick-Needles syndrome; frontometaphyseal dysplasia; filamin A

*Correspondence: Professor SP Robertson, Clinical Genetics Laboratory, Department of Paediatrics and Child Health, Dunedin School of Medicine, University of Otago, PO Box 913, Dunedin 9001, New Zealand. Tel: +643 479 7469; Fax: + 6434797469 ;

E-mail: stephen.robertson@stonebow.otago.ac.nz

Received 19 December 2005; revised 18 April 2006; accepted 21 April 2006; published online 23 August 2006

\section{Introduction}

The otopalatodigital spectrum disorders (including otopalatodigital syndrome type 1 and 2; OPD1, OPD2, frontometaphyseal dysplasia; FMD, Melnick-Needles syndrome, MNS) have long been considered to exhibit aetiological relatedness on account of their phenotypic similarities. ${ }^{1-4}$ Pedigree evidence and the more severe expression in males supports X-linked inheritance for the majority of cases. The skeletal dysplasia exhibited by these conditions consists of varying combinations and degrees of undertubulation of the long bones, cortical irregularity and campomelia. Thoracic hypoplasia can be associated and deafness (either conductive or sensorineural) is a common accompaniment. The more severe entities are associated with brain and visceral anomalies in the male (hydrocephalus, 


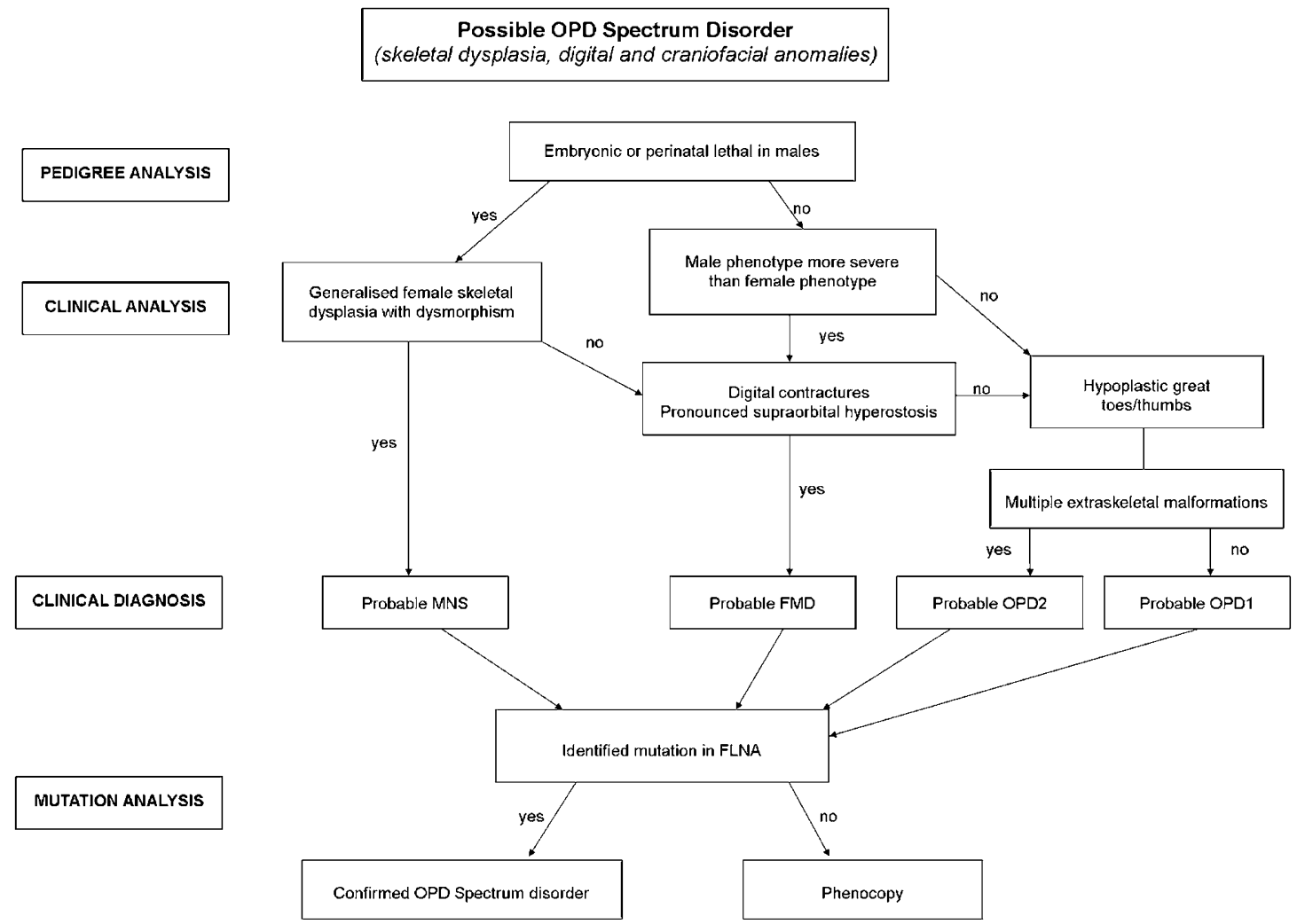

Figure 1 A diagnostic algorithm for the OPD spectrum disorders. As the clinical presentation of these conditions constitutes a spectrum, some phenotypes can exhibit overlap between diagnostic categories.

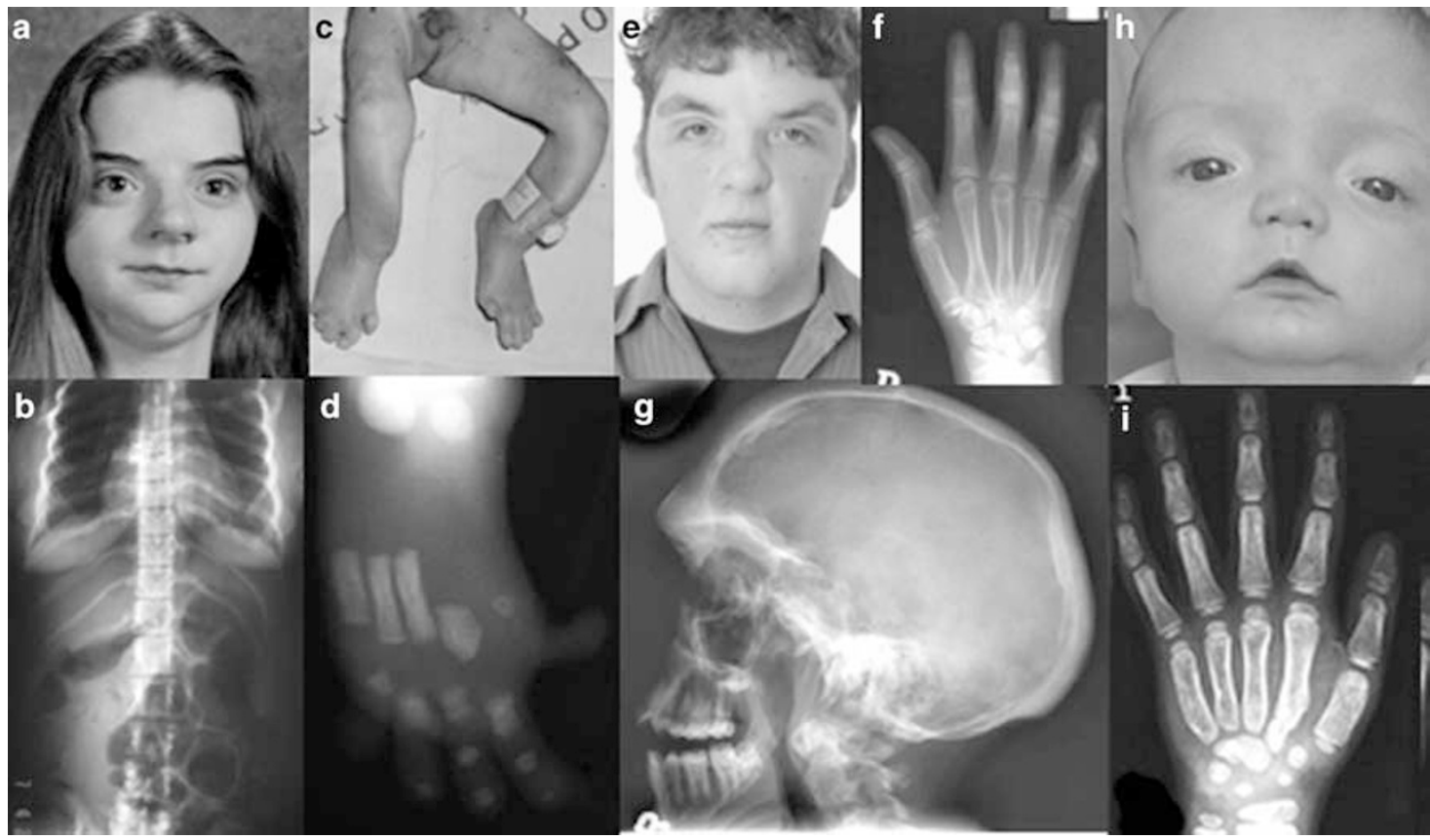

Figure 2 Clinical and radiological manifestions in the OPD spectrum disorders. (a) Facial appearance in MNS; (b) thoracic hypoplasia, irregular ribs and scoliosis in MNS; (c) campomelia and hypoplasia of the halluces in OPD2; (d) pattern defects in the foot of a male with OPD2; (e) facial features in FMD; (f) metacarpal and phalangeal modeling anomalies in FMD; (g) skull base sclerosis and supraorbital hyperostosis in FMD; (h) facies in OPD1; (i) metacarpal and phalangeal modeling anomalies in OPD1 including accessory epiphyseal ossification centre of metacarpal II. 
cerebellar hypoplasia, cardiac anomalies, omphalocoele and obstructive uropathy in OPD2 and MNS) ${ }^{4-6}$ with milder manifestations in males with OPD1 (cleft palate, hearing loss). ${ }^{7}$ The prevalence is unknown, but the conditions can confidently be described as rare $(<1$ in 100000 individuals).

\section{Clinical overview}

All four conditions exhibit phenotypic overlap but qualitative and quantitative differences make clinical distinction between the conditions useful (Figures 1 and 2).

\section{Otopalatodigital syndrome type 1}

OPD1 is the mildest phenotype in males of all four OPD spectrum disorders. ${ }^{7-10}$ Cardinal clinical features include, supraorbital hyperostosis, characteristic dysmorphism, deafness, pectus deformities and patterning anomalies of the digits (spatulate finger tips, short broad distal phalanx of the thumb, overlengthening of the second toe and foreshortening of the great toe). Females usually exhibit the facial dysmorphism more mildly, but occasionally can be as severely affected as males. ${ }^{11,12}$ Intellect is unimpaired. Stature is mildly reduced in most instances (Table 1).

\section{Otopalatodigital syndrome type 2}

The skeletal dysplasia in OPD2 is more severe than in OPD1.,13-16 The calvaria are hypomineralised in the neonatal period and there is pronounced skull base sclerosis. Thoracic hypoplasia, which can be life limiting, is common. Campomelia and patterning anomalies of the hands and feet are more pronounced than in OPD1 (eg absent hallux). Hydrocephalus, obstructive uropathy, cardiac defects and omphalocoele are recurrently observed. ${ }^{13,17} \mathrm{~A}$ majority die in the perinatal period or during infancy. Survivorship is associated with neurodevelopmental delay, but this is not invariable. ${ }^{16}$ Female carriers have a subclinical bone dysplasia and facial dysmorphism, but can occasionally manifest a more severe phenotype. ${ }^{18}$ Clinical discrimination from a female carrier with a mutation associated with OPD1 in a singleton female case is unreliable.

\section{Frontometaphyseal dysplasia}

The cardinal manifestations of FMD in the male are severe supraorbital hyperostosis, a skeletal dysplasia that includes campomelia, cortical irregularity and undertubulation of the long bones and deafness. ${ }^{19-21}$ Features defining it separately from OPD1, are more severe skeletal and facial manifestations, a relative lack of acral patterning defects (although distal phalangeal hypoplasia is often seen) and extraskeletal anomalies which include an asthenic build, metacarpophalangeal and interphalangeal joint contractures and malformations within the tracheobronchial tree. ${ }^{22-24}$ A lethal male variant has been described with

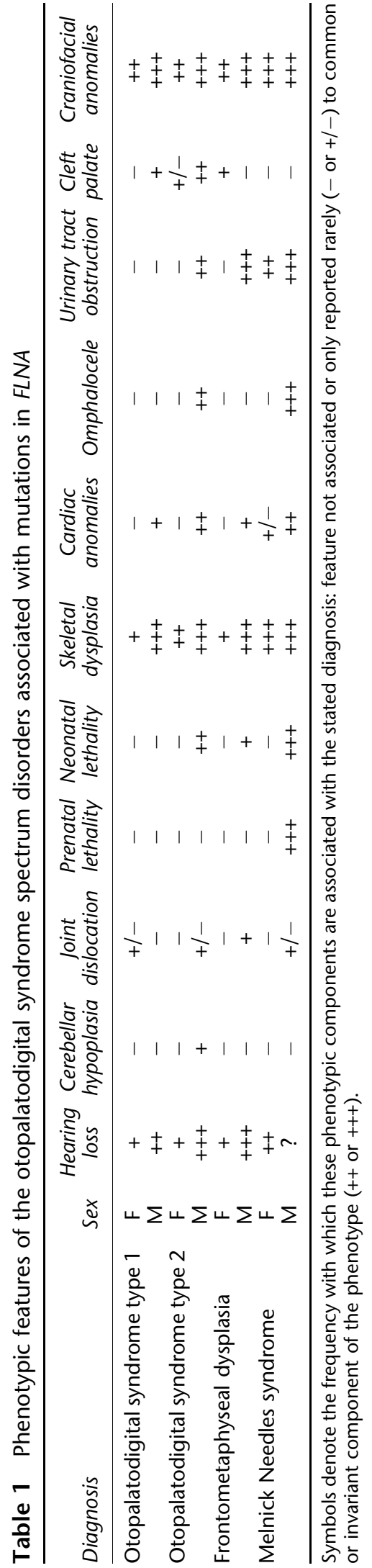




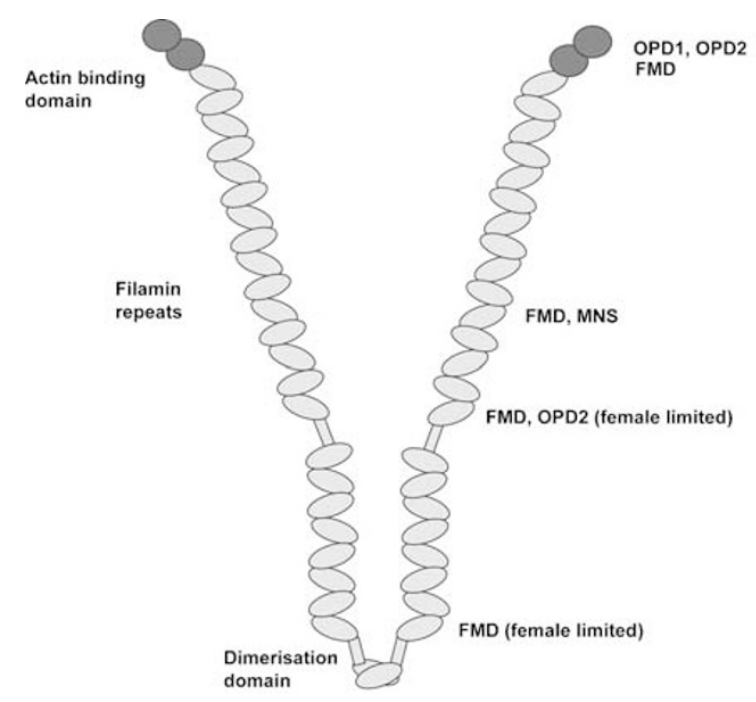

Figure 3 Diagram of a dimer of filamin A. The red regions represent the actin binding domains. There are 23 filamin repeats terminating in a 24th - the dimerisation domain. On the right the positions of the clustered substitutions and/or small in-frame deletions leading to the various OPD spectrum disorders are indicated.

obstructive uropathy and atrioventricular valve defects in addition to skeletal anomalies, contributing to perinatal demise of affected individuals. ${ }^{25}$

\section{Melnick-Needles syndrome}

This is almost an entirely female-limited entity due to the embryonic or perinatal lethality of almost all affected sons born to typically affected women. ${ }^{5,6}$ In males that survive to term, the phenotype is clinically indistinguishable from that associated with OPD2. ${ }^{4}$ Females have distinctive dysmorphism (supraorbital hyperostosis, exorbitism, full cheeks, micrognathia), thoracic hypoplasia due to short, irregular ribs, pronounced irregularity of the long bones, and long digits. ${ }^{26}$ Short stature is usually present, although exceptions have been reported. ${ }^{27}$ Intelligence is unimpaired. Occasionally MNS presents subclinically. ${ }^{27}$ Some individuals die in the second or third decade from respiratory failure. Urteric obstruction either at the pelvicaylceal or vesicoureteric junction is common. ${ }^{28}$ Some radiographic features are similar to those observed in Sphrintzen-Goldberg syndrome from which it can be clinically differentiated by the presence of craniosynostosis and mental retardation in the latter condition. ${ }^{29}$

\section{Overlap phenotypes}

Despite allelism between the discrete entities comprising the OPD spectrum and periventricular nodular heterotopia (PVNH), a neuronal migration disorder associated with epilepsy, mild cognitive disability but no skeletal dysplasia, only two convincing examples of overlap phenotypes between the two conditions have been reported. ${ }^{30} \mathrm{~A}$ sporadic female with a missense mutation in exon 45 demonstrated a combined PVNH-FMD phenotype, presumably on account of the mutation possessing the potential to promote aberrant splicing or alternatively lead to an amino-acid substitution. The second instance was a sporadic male with facial dysmorphism but no skeletal dysplasia in whom PVNH and an intestinal motility disorder were documented. ${ }^{31}$ The causative mutation produced a frameshift with subsequent downregulation of full-length FLNA transcription.

\section{Diagnostic approaches}

Determination of the correct diagnosis is most accurate upon consideration of the clinical presentation in the male. Variable expressivity in the female, determined in part by both the position of the underlying mutation and the effect of differing degrees of skewing of $\mathrm{X}$ inactivation, make prediction of the severity of the male phenotype from the presentation of an isolated female difficult. In the male, the marked differences in severity should lead to no problem in differentiating OPD1 from OPD2 or OPD2 from FMD although individuals and families have been described in whom overlap phenotypes clearly exist. ${ }^{1,2,25}$ Consideration of the female phenotype usually makes definition of OPD2 from MNS straightforward. ${ }^{4}$ Some have highlighted the close similarities between OPD1 and FMD in both males and females. ${ }^{3}$ In males, cleft palate is rare in FMD but not in OPD1. The extraskeletal anomalies noted in FMD may also assist in the differential diagnosis.

Clinical examination, full skeletal examination and ultrasound of the urinary tract are relevant first line investigations. Cerebral imaging and echocardiography may also assist where clinically indicated. Mutation analysis of the FLNA gene may also assist in prognostication given that many of the mutations underlying these disorders are recurrent and little interfamilial variability exists between unrelated males who have the same mutation. Molecular analysis can be staged, with initial focus given to the region of the gene implicated by the clinical diagnosis (Figure 3).

\section{Molecular and clinical basis of the diseases}

The vast majority of familial cases exhibit X-linked inheritance. Marked manifestations in the female, particularly for frontometaphyseal dysplasia make autosomal dominant inheritance in some instances a formal possibility although male-to-male transmission has never been observed. Families exhibiting consanguinity and sib recurrence of an OPD2-like disorder have been observed suggesting that an autosomal recessive phenocopy of this condition exists.

\section{Mutations in FLNA}

The only locus associated to date with the OPD spectrum disorders is the gene, FLNA, encoding the cytoskeletal 
protein, filamin A. FLNA comprises 48 exons and encodes a modular protein with an N-terminal actin-binding domain and a tail of 24 structurally homologous repeats. This repeat region is interspaced by two hinge regions between repeats 15 and 16 and 23 and 24, and terminates in a dimerisation domain (repeat 24). All three filamin paralogues (filamin A, filamin B and filamin C) share this same modular organisation and share considerable homology at the secondary structure level. Filamin A forms homodimers in vivo that bind actin through $\mathrm{N}$-terminal actin-binding domains (Figure 3). A multiplicity of other interacting proteins bind filamin through tandemly arranged filamin repeats. Cellular functions mediated by filamin include linking signal transduction events to the modulation of the actin cytoskeleton and gene transcription. ${ }^{32}$

All instances of OPD1 and MNS so far reported in the literature have had mutations observed in FLNA. ${ }^{33}$ For OPD2 and FMD the proportion reported with a FLNA mutation in one series was 70 and $43 \%$, respectively. ${ }^{33}$ All mutations underlying the OPD-spectrum disorders are missense or small deletions that preserve the translational reading frame. Immunostaining and Western blot analysis of cells obtained from males with identified mutations indicate that full-length filamin A, that retains its association with the actin cytoskeleton, is still produced. This mutational mechanism distinguishes the mode of pathogenesis for the OPD spectrum disorders from $\mathrm{X}$-linked PVNH, which is associated with loss-of-function alleles and lack of filamin A expression in cells expressing the mutant allele. ${ }^{34,35}$

The mutations that lead to all three disorders are clustered over the gene and exhibit a genotype-phenotype correlation. $^{33}$ All cases of typical OPD1 and OPD2 with identifiable mutations have predicted substitutions in the actin-binding domain. Three females with an OPD2-like phenotype had disease-associated substitutions in the rod domain of filamin A. There was no male relative to demonstrate the corresponding male phenotype in all three instances, and consequently it is reasonable to view the diagnosis of OPD2 in these families as provisional. All individuals with MNS $(n=12)$ had mutations leading to one of only three mutations in exon 22 (encoding filamin repeat 10) associated with this diagnosis. Mutations underlying FMD were the most dispersed - found in exons encoding substitutions in repeats $10,14-15$ and 23. A recent study examining an extended cohort of individuals with the diagnosis, identified mutations in $57 \%$ of individuals, reinforcing an impression that this condition is characterized by locus heterogeneity. No consistent clinical feature differentiates those patients in whom FLNA mutations are identifiable from those who have no such demonstrable filamin A defect.

Variability in skewing of $\mathrm{X}$-inactivation in females has been shown to correlate with the severity of the OPD spectrum phenotypic categories. ${ }^{18,33}$ Milder phenotypes (ie OPD1) are associated with more random skewing of $\mathrm{X}$ inactivation; females with OPD2 or MNS have near complete skewing favouring expression of the nonmutant allele. In individual cases the degree of skewing does not correlate with the severity of the associated phenotype, an observation that may be accounted for by the presence of mosaicism or tissue or age-related variability in the inactivation process. This observation, together with the potential for locus heterogeneity for some of these conditions, cautions against the use of $\mathrm{X}$ inactivation analysis as a guide for the severity of a male phenotype upon presentation of an isolated affected male.

\section{Mosaicism \\ Both somatic and germline mosaicism have been observed in FLNA leading to PVNH and have been associated with modulation of the severity of the phenotype and survivor- ship in males. ${ }^{36,37}$ Germline mosaicism has been observed in association with OPD1 and a somatic mutation has been shown to have led to MNS, suggesting similar processes may modulate the expression of OPD spectrum phenotypes. $^{38}$}

\section{Management Genetic counselling}

Accurate risk assessment in families is dependent on assigning the correct diagnosis, which is best defined from the male phenotype. Variable expressivity in the female can make prediction of the severity of the male phenotype difficult and unreliable. Although all typical instances of OPD1 and MNS have been associated with FLNA mutations, a sizable fraction of male and female cases with FMD and an unknown fraction of individuals with OPD2 have no identifiable FLNA mutation. In this instance of FMD, no male-to-male transmission of the phenotype has been reported, but instances of vertical transmission from mother-to-daughter in the absence of demonstrable mutation in FLNA, leaves open the possibility of autosomal dominant inheritance. An autosomal recessive condition has been reported as an instance of OPD1, but the digital malformations are distinct, and as such it does not represent a true phenocopy of the disorder. ${ }^{39}$

Germline mosaicism has been documented for OPD1 and therefore the risk of sib recurrence when the mother has tested negative for the causative FLNA mutation is higher than the background new mutation rate.

\section{Genetic testing strategy}

Sequence analysis of FLNA can initially focus on the established regions where mutations have been shown to cluster (exons 3-5, 11, 22, 27-29 and 45). ${ }^{33}$ With the description of more mutations underlying the OPD spectrum disorders, these mutation hotspots may be widened further. Directed mutation searching can be 
initially guided by the underlying clinical diagnosis. To date, all mutations causative of OPD1 have been located in exon 3-5, encoding the actin-binding domain of filamin A. Similarly all instances of classically affected males with OPD2 have mutations in the same region. Some families with overlap phenotypes between OPD1 and OPD2 also have exon 3-5 mutations. ${ }^{2}$ A small number of females with a severe, OPD2-like phenotype have been described with mutations in exons 11 and $29 .{ }^{33}$ Inference from observations of male lethality in MNS suggest that these mutations may confer a severe, possibly embryonic lethal, phenotype in the hemizygous state.

The mutational spectrum underlying MNS is very narrow. All 12 unrelated females with this condition had one of three mutations leading to this condition; two of these mutations being highly recurrent. ${ }^{33}$ It is therefore reasonable to restrict initial mutation screening for this condition to exon 22 where all of these mutations are located. Mutational analysis of a male affected with MNS has yet to be reported.

Mutations underlying FMD are dispersed the most widely over the FLNA locus. These mutations are still clustered, within exons $4,22,29,33$, and $44-46 .^{25,30,33}$ The typical male phenotype is associated with the more $5^{\prime}$ mutations; only females have been reported with mutations in the most $3^{\prime}$ hotspot, some of them with cleft palate which is an uncommon manifestation in FMD. A severe perinatal-lethal phenotype has recently been described in association with mutations in exons 22 and $29 .^{25}$

\section{Complications}

Hearing loss Sensorineural or conductive or combined forms of hearing loss are common accompaniments of all OPD spectrum disorders. Losses are often only moderate in degree and consequently late diagnosis is a frequent occurrence.

Scoliosis Progressive scoliosis is well documented in FMD and MNS, commonly requiring surgical intervention. ${ }^{40-42}$ This complication must be monitored for in heterozygous females with FMD regardless of the severity of other manifestations of the condition.

Restrictive and obstructive lung disease are also associations, not only due to thoracic hypoplasia and co-existent scoliosis, but also abnormalities within the tracheobronchial tree. ${ }^{22,24}$

Urinary tract obstruction Males with FMD often present with urethral and/or ureteric obstruction most frequently at the vesicoureteric junction. ${ }^{23}$ Females with MNS can present with hydronephrosis secondary to pelvicalyceal junction obstruction. ${ }^{28}$

Neurodevelopment and psychological aspects Intelligence is unimpaired in OPD1, FMD and females with
MNS. Some surviving individuals with OPD2 have been documented to have global delay in neurodevelopment. The significant craniofacial manifestations of the OPD spectrum disorders often precipitate significant psychological morbidity, prompting some to seek craniofacial surgery to ameliorate the cosmetic effects of the condition. ${ }^{43}$

\section{Prenatal diagnosis}

In the presence of a known FLNA mutation, prenatal diagnosis via CVS or amniocentesis is possible. Ultrasound scanning can detect some of the anomalies associated with these phenotypes such as thoracic hypoplasia, omphalocoele or hydronephrosis. ${ }^{44}$

\section{Conclusion}

The multitude of conditions that are caused by mutations in the genes encoding the cytoskeletal proteins, the filamins, ${ }^{33,34,45-47}$ indicate that they play a critical link in organogenesis in multiple tissues. The clustered distribution of mutations over FLNA leading to a spectrum of conditions in male hemizygotes suggests that filamin $\mathrm{A}$ is a protein with multiple modular functions. ${ }^{48}$ The variable expressivity in females, however, emphasizes that not only genetic but epigenetic influences impact upon phenotypic expression in the heterozygote. Further insights will arrive when the identity of the pathways mediated by filamins during organogenesis are better defined and delineated.

\section{Acknowledgements}

The author thanks the families and individuals who have contributed to our work on these conditions and their referring clinicians. Thanks to Drs Y Alanay, S Aftimos, S Manouvrier-Hanu and M Whiteford for assistance with Figure 2. The author is supported by the Child Health Research Foundation of New Zealand.

\section{References}

1 Verloes A, Lesenfants S, Barr M et al: Fronto-otopalatodigital osteodysplasia: clinical evidence for a single entity encompassing Melnick-Needles syndrome, otopalatodigital syndrome types 1 and 2, and frontometaphyseal dysplasia. Am J Med Genet 2000; 90: 407-422.

2 Horn D, Nitz I, Bollmann R: Oto-palato-digital syndrome with features of type I and II in brothers. Genet Couns 1995; 6: 233-240.

3 Gimelli F, Superti-Furga A: Frontometaphyseal dysplasia and the oto-palato-digital syndrome. Dysmorphol Clin Genet 1987; 1: 2-5.

4 Robertson S, Gunn T, Allen B, Chapman C, Becroft D: Are Melnick-Needles syndrome and oto-palato-digital syndrome type II allelic? Observations in a four-generation kindred. Am J Med Genet 1997; 71: 341-347.

5 Donnenfeld AE, Conard KA, Roberts NS, Borns PF, Zackai EH: Melnick-Needles syndrome in males: a lethal multiple congenital anomalies syndrome. Am J Med Genet 1987; 27: 159-173.

6 Krajewska-Walasek M, Winkielman J, Gorlin RJ: Melnick-Needles syndrome in males. Am J Med Genet 1987; 27: 153-158.

7 Dudding BA, Gorlin RJ, Langer LO: The oto-palato-digital syndrome. A new symptom-complex consisting of deafness, 
dwarfism, cleft palate, characteristic facies, and a generalized bone dysplasia. Am J Dis Child 1967; 113: 214-221.

8 Le Marec B, Odent S, Bracq E, Bulard MB, Bourdiniere J, Babut JM: [Oto-palato-digital type I syndrome in five generations. Relationship to the type II form]. Ann Genet 1988; 31: $155-161$.

9 Podoshin L, Heymans HS, Fradis M: The oto-palato-digital syndrome. J Laryngol Otol 1976; 90: 407-411.

10 Buran DJ, Duvall III AJ: The oto-palato-digital (OPD) syndrome. Arch Otolaryngol 1967; 85: 394-399.

11 Gorlin RJ, Poznanski AK, Hendon I: The oto-palato-digital (OPD) syndrome in females. Oral Surg Oral Med Oral Pathol 1973; 35: 218-224.

12 Langer Jr LO: The roentgenographic features of the oto-palatodigital (OPD) syndrome. Am J Roentgenol Radium Ther Nucl Med 1967; 100: 63-70.

13 Young K, Barth CK, Moore C, Weaver DD: Otopalatodigital syndrome type II associated with omphalocele: report of three cases. Am J Med Genet 1993; 45: 481-487.

14 Fitch N, Jequier S, Gorlin R: The oto-palato-digital syndrome, proposed type II. Am J Med Genet 1983; 15: 655-664.

15 Fitch N, Jequier S, Papageorgiou A: A familial syndrome of cranial, facial, oral and limb anomalies. Clin Genet 1976; 10: 226-231.

16 Andre M, Vigneron J, Didier F: Abnormal facies, cleft palate, and generalized dysostosis: a lethal X-linked syndrome. J Pediatr 1981; 98: $747-752$.

17 Stratton RF, Bluestone DL: Oto-palatal-digital syndrome type II with X-linked cerebellar hypoplasia/hydrocephalus. Am J Med Genet 1991; 41: 169-172.

18 Robertson SP, Walsh S, Oldridge M, Gunn T, Becroft D, Wilkie AO: Linkage of otopalatodigital syndrome type 2 (OPD2) to distal Xq28: evidence for allelism with OPD1. Am J Hum Genet 2001; 69: 223-227.

19 Gorlin RJ, Cohen Jr MM: Frontometaphyseal dysplasia. A new syndrome. Am J Dis Child 1969; 118: 487-494.

20 Danks DM, Mayne V: Frontometaphyseal dysplasia: a progressive disease of bone and connective tissue. Birth Defects Orig Artic Ser 1974; 10: 57-60.

21 Holt JF, Thompson GR, Arenberg IK: Frontometaphyseal dysplasia. Radiol Clin North Am 1972; 10: 225-243.

22 Leggett JM: Laryngo-tracheal stenosis in frontometaphyseal dysplasia. J Laryngol Otol 1988; 102: 74-78.

23 Kanemura T, Orii T, Ohtani M: Frontometaphyseal dysplasia with congenital urinary tract malformations. Clin Genet 1979; 16: 399-404.

24 Fitzsimmons JS, Fitzsimmons EM, Barrow M, Gilbert GB: Frontometaphyseal dysplasia. Further delineation of the clinical syndrome. Clin Genet 1982; 22: 195-205.

25 Stefanova M, Meinecke P, Gal A, Bolz H: A novel 9 bp deletion in the filamin a gene causes an otopalatodigital-spectrum disorder with a variable, intermediate phenotype. Am J Med Genet A 2005; 132: 386-390.

26 Melnick JC, Needles CF: An undiagnosed bone dysplasia. A 2 family study of 4 generations and 3 generations. Am J Roentgenol Radium Ther Nucl Med 1966; 97: 39-48.

27 Kristiansen M, Knudsen GP, Soyland A, Westvik J, Orstavik KH: Phenotypic variation in Melnick-Needles syndrome is not reflected in $\mathrm{X}$ inactivation patterns from blood or buccal smear. Am J Med Genet 2002; 108: 120-127.

28 LaMontagne AE: Urological manifestations of the MelnickNeedles syndrome: a case report and review of the literature. J Urol 1991; 145: 1020-1021.
29 Greally MT, Carey JC, Milewicz DM et al: Shprintzen-Goldberg syndrome: a clinical analysis. Am J Med Genet 1998; 76: 202-212.

30 Zenker M, Rauch A, Winterpacht A et al: A dual phenotype of periventricular nodular heterotopia and frontometaphyseal dysplasia in one patient caused by a single FLNA mutation leading to two functionally different aberrant transcripts. Am J Hum Genet 2004; 74: 731-737.

31 Hehr U, Hehr A, Uyanik G, Phelan E, Winkler J, Reardon W: A Filamin A splice mutation resulting in a syndrome of facial dysmorphism, periventricular nodular heterotopia and severe constipation reminiscent of Cerebro-Fronto-Facial syndrome. J Med Genet 2005 doi:10.1136/jmg.2005.038505.

32 Stossel TP, Condeelis J, Cooley L et al: Filamins as integrators of cell mechanics and signalling. Nat Rev Mol Cell Biol 2001; 2: 138-145.

33 Robertson SP, Twigg SR, Sutherland-Smith AJ et al: Localized mutations in the gene encoding the cytoskeletal protein filamin A cause diverse malformations in humans. Nat Genet 2003; 33: $487-491$.

34 Fox JW, Lamperti ED, Eksioglu YZ et al: Mutations in filamin 1 prevent migration of cerebral cortical neurons in human periventricular heterotopia. Neuron 1998; 21: 1315-1325.

35 Sheen VL, Dixon PH, Fox JW et al: Mutations in the X-linked filamin 1 gene cause periventricular nodular heterotopia in males as well as in females. Hum Mol Genet 2001; 10: 1775-1783.

36 Guerrini R, Mei D, Sisodiya S et al: Germline and mosaic mutations of FLN1 in men with periventricular heterotopia. Neurology 2004; 63: 51-56.

37 Parrini E, Mei D, Wright M, Dorn T, Guerrini R: Mosaic mutations of the FLN1 gene cause a mild phenotype in patients with periventricular heterotopia. Neurogenetics 2004; 5: 191-196.

38 Robertson SP, Thompson S, Morgan T et al: Postzygotic mutation and germline mosaicism in the otopalatodigital syndrome spectrum disorders. Eur J Hum Genet 2006; 14: 549-554

39 Zaytoun GM, Harboyan G, Kabalan W: The oto-palato-digital syndrome: variable clinical expressions. Otolaryngol Head Neck Surg 2002; 126: 129-140.

40 Morava E, Illes T, Weisenbach J, Karteszi J, Kosztolanyi G: Clinical and genetic heterogeneity in frontometaphyseal dysplasia: severe progressive scoliosis in two families. Am J Med Genet A 2003; 116: 272-277.

41 Medlar RC, Crawford AH: Frontometaphyseal dysplasia presenting as scoliosis. J Bone Joint Surg Am 1978; 60: 392-394.

42 Bartolozzi P, Calabrese C, Falcini F, Giovannucci Uzzielli ML, Maggini M: Melnick-Needles syndrome: osteodysplasty with kyphoscoliosis. J Pediatr Orthop 1983; 3: 387-391.

43 Kung DS, Sloan GM: Cranioplasty in frontometaphyseal dysplasia. Plast Reconstr Surg 1998; 102: 1144-1146.

44 Eccles DM, Moore IE, Cook S et al: Prenatal ultrasound findings in a fetus with otopalatodigital syndrome type II. Clin Dysmorphol 1994; 3: 175-179.

45 Krakow D, Robertson SP, King LM et al: Mutations in the gene encoding filamin B disrupt vertebral segmentation, joint formation and skeletogenesis. Nat Genet 2004; 36: 405-410.

46 Sheen VL, Jansen A, Chen MH et al: Filamin A mutations cause periventricular heterotopia with Ehlers-Danlos syndrome. Neurology 2005; 64: 254-262.

47 Vorgerd M, van der Ven PF, Bruchertseifer V et al: A mutation in the dimerization domain of filamin c causes a novel type of autosomal dominant myofibrillar myopathy. Am J Hum Genet 2005; 77: 297-304.

48 Robertson SP: Filamin A: phenotypic diversity. Curr Opin Genet Dev 2005; 15: 301-307. 